\title{
Tecnura
}

\section{Diagnóstico de los efectos generados por el tráfico de largo destino en la malla vial del municipio de Cachipay, Cundinamarca}

\section{Diagnosis of the effects generated by long-distance traffic in the road network of the municipality of Cachipay, Cundinamarca}

\section{Espinel Duarte Luis Eduardoํㅜ, Ladino Chaves Oswaldo ${ }^{2}$, Iguarán Salinas Lauren Diane ${ }^{3}$}

Fecha de recepción: 22 de noviembre de 2017

Fecha de aceptación: 9 de marzo de 2018

Cómo citar: Espinel D., L.E., Ladino C., O. e Iguarán S., L.D. (2018). Diagnóstico de los efectos generados por el tráfico de largo destino en la malla vial del municipio de Cachipay. Revista Tecnura, 22(56), 62-75. DOI: https:// doi.org/10.14483/22487638.13761

\section{Resumen}

Contexto: Este estudio presenta la débil infraestructura que existe en las carreteras de largo destino. Se muestra, como estudio de caso, el municipio de Cachipay el cual no escapa a una serie de inconvenientes en materia de movilidad debido a las fallas de la malla vial.

Método: La investigación se desarrolló en la línea de investigación de la modelación y simulación de problemas; con el planteamiento de un estudio de caso mediante el análisis e inventario de daños, por medio de dos alternativas, escogiendo la más apropiada.

Resultados: La búsqueda de soluciones a los problemas de movilidad en el municipio de Cachipay ha generado un incremento de circulación vehicular por las calles del municipio, que oscila entre un $67 \%$ a un $46 \%$, según los conteos de tráfico registrados en los días entre semana contra días festivos. Conclusiones: Cachipay, por ser un municipio cercano a la capital, debe contar con propuestas que contribuyan a solucionar el deterioro de las calles y vías alternas, y a fortalecer el parque automotor del municipio.

Palabras clave: Cachipay, inventario de daños, malla vial, movilidad vehicular, tráfico de largo alcance.

\begin{abstract}
:
Context: This study presents the weak infrastructure that exists on long-haul roads. The municipality of Cachipay, Cundinamarca, is taken as a case study, which does not escape a series of mobility inconveniences due to the failures of the road network.

Method: The research was developed in the line of investigation of modeling and simulation of problems, with the approach of a case study through inventory and damage analysis, developing two alternatives, and choosing the most appropriate one.

Results: The search for solutions to mobility problems in the municipality of Cachipay has generated an increase in vehicular traffic through the streets of the municipality, ranging from $67 \%$ to $46 \%$, according
\end{abstract}

1 Ingeniero Civil, especialista en Diseño y Construcción de Vías y Aeropistas, candidato a magíster en Infraestructura Vial. Director de obra Latinco S.A. Bogotá, Colombia. Contacto: ingeniero1espinel@ hotmail.com

2 Ingeniero Civil, especialista en Diseño y Construcción de Vías y Aeropistas, especialista en Gerencia de Proyectos, candidato a magíster en Infraestructura Vial, director de Proyectos en Silva Carreño y Asociados S.A.S. Bogotá, Colombia. Contacto: oslacha@gmail.com

3 Directora del proyecto, ingeniera Civil, magíster en Ingeniería Civil, MBA. Líder del equipo de Supervisión Agencia Nacional de Infraestructura. Bogotá, Colombia. Contacto: lauren_iguaran@hotmail.com 
to traffic counts recorded on weekdays against the holidays.

Conclusions: Because it is a municipality near the capital, Cachipay ought to have proposals that contribute to solve the deterioration of the streets and alternate roads, and to strengthen the municipal vehicle fleet.

Keywords: Cachipay, damage inventory, road network, vehicular mobility, Long-range traffic.

\section{Introducción}

El Estado colombiano, con el fin de agilizar la movilidad, ha implementado planes retorno en todo el territorio Nacional, que inicialmente solo eran para los puentes festivos, pero se han extendido para fines de semana normales. Por otra parte, se han convertido, de alguna manera, en rutas permanentes y agradables para los turistas, gracias a los atractivos que ofrecen las regiones: flores, frutas, artesanías, gastronomía, clima, etc. En ese sentido, se ha incrementado el paso de vehículos de largo destino por pequeños municipios que no cuentan con una infraestructura vial diseñada e implementada en sus planes de ordenamiento territorial (POT) que permita soportar un elevado número de repeticiones de carga.

Uno de estos planes retorno es el par vial que implementa el Ministerio de Obras Públicas y Transporte, entre el municipio de La Mesa (Cundinamarca) y Bogotá D.C., cuyo flujo vehicular, en el sentido Bogotá-La Mesa, es desviado por las poblaciones de Bogotá-Mosquera-Madrid-Cartagenita (Facatativá)-Zipacón-Cachipay-La Gran Vía-Tena.

Al respecto, es importante establecer que la problemática radica en la infraestructura vial de Cachipay, la cual se desarrolló en torno a tres ejes viales significativos: vía Bogotá-La Mesa, vía Bojacá-Peña Negra y la vía Férrea Bogotá-Girardot, que hacen de su distribución e implantación urbana algo muy orgánico, y causan algunos obstáculos en la circulación y movilidad vial.

Muchos problemas de movilidad intermunicipal se presentan porque no existen horarios estrictamente establecidos en las empresas de transporte; no se tienen espacios adecuados ni suficientes para el peatón, no existe el control de velocidad tanto en el sector rural como en el urbano, hay un déficit en el sistema de señalización y movilidad del tránsito vehicular y peatonal, existe un gran desorden en el sistema de bahías de parqueo en el casco urbano e infracciones de tránsito. (Concejo Municipal, 2008)

Adicionalmente, Cachipay presenta grandes problemas de movilización por la falta de mantenimiento y terminación de vías, como es el caso de los 7 km existentes para llegar a la inspección de Peña Negra, y muchas otras que se conectan con las regiones vecinas y que a su vez permiten el intercambio de productos de una a otra región del municipio.

En total el municipio cuenta con sesenta y cuatro (64) km de vía terciaria sin pavimentar, dieciséis y medio $(16,5) \mathrm{km}$ de vía terciaria pavimentada y aproximadamente cuatro (4) km de vías urbanas de las cuales un $80 \%$ de las mismas se encuentran en mal estado. (Concejo Municipal, 2008)

Dentro de este contexto, la implementación de los planes de retorno para agilizar la movilidad de los corredores viales nacionales ha ocasionado la desviación de un alto volumen de tráfico vehicular compuesto por todo tipo de vehículos livianos, buses, camiones y tractomulas. Esto, a su vez, ha generado un alto grado de deterioro en las vías internas de los municipios, incrementos en los índices de accidentalidad, afectaciones en la movilidad, incremento en los niveles de ruido y polución, afectaciones a la comodidad, a la economía y el confort de los locales y de los turistas en los municipios.

El municipio de Cachipay no es ajeno a esta problemática por el paso del tráfico de largo destino, generado por la implementación de una ruta vehicular sentido Bogotá-Cachipay. Así, las vías internas del municipio presentan una serie de daños como: 
- Fisuras: longitudinales y transversales en juntas de construcción, por reflexión de juntas o grietas en placas de concreto, en medialuna, de borde, en bloque, piel de cocodrilo, por deslizamiento de capas y fisuración incipiente.

- Deformaciones: ondulaciones, abultamientos, hundimientos y ahuellamiento.

- Pérdida de las capas de la estructura: descascaramientos, baches y parches.

- Daños superficiales: desgastes superficiales, pérdida de agregado, exudación y surcos.

- Otros: afloramiento de finos y afloramiento de agua.

Todos estos daños afectan la movilidad vehicular en el municipio. Adicionalmente, se presenta afectación las zonas de circulación peatonal; razón por la cual, tal deterioro exige una pronta solución acorde con lo propuesto por el gobierno, en la utilización de las vías de Cachipay que en un comienzo se habilitaron para el plan retorno, pero que en la actualidad son paso obligado de los vehículos que van hacia los municipios aledaños, departamentos o la capital.

En ese sentido, la eficiencia de los medios de transporte define en gran medida la competitividad de una región, "la cual se traduce en una mayor circulación de bienes y servicios y a su vez en un menor costo de fletes y demás factores relacionados directa e indirectamente sobre el valor y disponibilidad de los productos ofrecidos y demandados" (Gobernación de Cundinamarca, 2009, p. 88).

En este contexto, "una región que posee modos de transporte eficientes tendrá en el actual mercado global una mayor ventaja competitiva" (Gobernación de Cundinamarca, 2009, p. 89). Pues, dentro de la descripción de la red vial del departamento de Cundinamarca, al que pertenece el municipio de Cachipay, se encuentra que este forma parte de la red vial secundaria que tiene una longitud total de

[...] 4997 kilómetros, de los cuales 1670 kilómetros están en pavimento y 3297 kilómetros están en material de afirmado y 30 kilómetros están en tierra. Del total de la red secundaria se puede analizar que el $33,4 \%$ se encuentra en pavimento y el $66,0 \%$ en afirmado. (Gobernación de Cundinamarca, 2009, p. 94)

Como se puede observar, el mayor porcentaje de kilómetros de la red vial departamental es el afirmado, situación que afecta la integración, conectividad, productividad y agroturismo en aquellas provincias donde se concentran dichos tramos viales, a los cuales esporádicamente se les realiza mantenimiento rutinario, sobre todo en épocas de verano, originando incomunicación entre los municipios del departamento y de estos con las arterias principales.

La red secundaria favorece el desarrollo económico en aquellos municipios donde no se tiene acceso directo a las carreteras principales, pero que generan un gran porcentaje de la producción agrícola del departamento, tal es el caso de los municipios ubicados en las provincias especialmente de Sumapaz, Medina y Rionegro. (Gobernación de Cundinamarca, 2009, p. 88).

De modo que, dentro de la descripción de la red vial secundaria a cargo del departamento de Cundinamarca, la situación del municipio de Cachipay se detalla en la tabla 1.

Tabla 1. Red vial secundaria - Cundinamarca / Cachipay

\begin{tabular}{llcccc}
\hline Código de la vía & \multicolumn{1}{c}{ Tramo desde/hasta } & Pavimentado & Afirmado & Tierra & Longitud total \\
\hline 47CN11 & La Gran Vía-Cachipay-Petaluma-Anolaima-Corralejas & 35 & 0 & 0 & 35 \\
\hline 47CN11-3-1 & Cachipay-Peña Negra-Anolaima & 2 & 17 & 0 & 19 \\
\hline & Cachipay-Calandaima-Anolaima & 3 & 2 & 0 & 5 \\
\hline TOTAL & & 40 & 19 & 0 & 59 \\
\hline
\end{tabular}

Fuente: Gobernación de Cundinamarca (2009, p. 88). 
A cargo del Instituto Nacional de Vías (Invías) no hay en la actualidad ninguna vía de Cachipay. Así, de lo expuesto se deduce que las vías a cargo del departamento se encuentran en buen estado; sin embargo, se debe tener en cuenta que estas no están pavimentadas, aspecto fundamental para desarrollo del proyecto que antecede el presente artículo; pues independientemente que estén o no en buen estado, las vías deben estar todas pavimentadas, máximo si se tiene en cuenta que se han convertido en vías de alto flujo vehicular. De otra parte, resulta trascendental hacer alusión a los 67 kilómetros sin pavimentar a cargo del municipio, los cuales se encuentran en regular y mal estado, lo que significa que se hace indispensable una propuesta conducente a solucionar esta situación, que puede partir de una iniciativa que integre a Invías.

Para lo anterior, se debe considerar que la movilidad ha generado un espacio de disertaciones académicas que buscan comprender lo que significa el desplazamiento de las personas, con ocasión de sus diferentes actividades dentro y fuera de la ciudad. De este modo, la movilidad se ve reflejada en el consumo de espacio ocasionado por las personas y toda clase de vehículo -automóviles, buses, motos, camiones, ciclas, entre otros-, hecho que genera una serie de problemáticas sociales, económicas y ambientales relacionadas con el desplazamiento de sus habitantes.

Por consiguiente, el uso de diversas formas de transporte "está frecuentemente influenciado por el nivel de ingreso. Personas con bajos ingresos desempeñan mucho más el papel de peatones, ciclistas y usuarios de transporte público; personas con ingresos más altos suelen desempeñarse como motoristas o pasajeros de automóviles" (Alcántara, 2010, p. 36). Así, la circulación de personas tiene diversas consecuencias para quienes habitan un mismo ambiente, entre ellas: el consumo de recursos y los impactos ambientales.

El primero integra el consumo de distintos bienes inmateriales y materiales. El inmaterial hace referencia "al tiempo de desplazamiento, ya que este es un recurso escaso para todos. Disminuir el consumo de tiempo al mínimo posible siempre es el objetivo final para que quienes se desplazan puedan usarlo también en la realización de otras actividades" (Alcántara, 2010, p. 51). El consumo material se orienta al espacio que requiere la construcción de "aceras, autopistas, terminales de autobuses, estaciones de trenes y ferrocarriles metropolitanos, a los que se suman las personas, la energía y los recursos financieros" (p. 51).

Como se observa, el factor inmaterial resulta determinante en la movilidad, debido a que, de este depende el tiempo que las personas necesitan para desplazarse de un lado a otro, el cual se ve afectado por el número de personas y por cómo está construida la ciudad, de cuya construcción emergen anomalías que repercuten en los tiempos de desplazamiento. Este hecho, día tras día, es más complejo, debido al número de habitantes que tiene Colombia.

En cuanto a los impactos ambientales, estos se clasifican en el uso de los recursos escasos o raros,

[...] como el hierro, el látex y el plástico utilizados para construir vehículos, y los minerales, piedras y asfalto, para pavimentar caminos. Lo desechos de materiales como automóviles viejos, aceites lubricantes, neumáticos y los relacionados a las externalidades que hacen referencia a los accidentes por el tráfico vehicular, la contaminación del aire, el ruido y la congestión. (Alcántara, 2010, p. 52).

Un derrotero de la movilidad lo constituyen los impactos ambientales, que con el pasar del tiempo se han multiplicado; y que también, por ser una constante en la vida de los seres humanos, requieren de una especial atención. En este sentido, los retos y apuestas para la región Centro Oriente al 2018 es "descongestionar y articular la red primaria de transporte y desconcentrar centros logísticos, que potencien la productividad y la infraestructura estratégica" (Gaviria, 2016, p. 13), teniendo en cuenta que de los recursos del plan plurianual de inversiones 2015-2108 sobresale el sector del transporte. De ese modo, 
[...] un pilar para construir ventajas competitivas y aprovechar las ventajas comparativas de Cundinamarca está en la infraestructura y los sistemas de transporte y movilidad. En este escenario, el departamento cuenta con aproximadamente $21.164 \mathrm{~km}$ de carreteras de los cuales 890 corresponden a la red nacional o primaria, 6.881 a la red secundaria y 11.758 de la red terciaria. (Rey, 2016, p. 133)

Además, hay que considerar que uno de los problemas que más afecta a la competitividad del departamento es su elevado porcentaje de vías, que se encuentran en regular o mal estado. Del total de la red vial departamental, solo $13 \%$ se encuentra "pavimentado y el $87 \%$ en afirmado. De la malla vial, el $40 \%$ se encuentra en mal estado, el $30 \%$ presenta un regular estado y el $30 \%$ restante está en buen estado, principalmente en las vías de la sabana de Bogotá" (Rey, 2016, p. 136). Por tanto, la actual malla vial es insuficiente para atender la demanda de carga y pasajeros que deben movilizarse por el territorio cundinamarqués.

La infraestructura vial es de vital importancia en el desarrollo y crecimiento de un país, mueve la economía y vincula las regiones aisladas.

[...] [Además,] la infraestructura vial de cualquier país del mundo es diseñada para incorporar todo el territorio a la economía nacional, trenes, carreteras y aeropuertos, tienen este fin y cumplen un papel fundamental, en el desarrollo y crecimiento económico de los países. (Zamora, 2012, p. 16)

Cabe anotar que en la actualidad la inversión por parte del Estado colombiano en la mejora de la infraestructura vial

[...] es un tema de gran importancia para la economía y la sociedad en general; por lo tanto, Colombia presenta un rezago significativo en lo referente al desarrollo de las vías terrestres nacionales, cuyo progreso a lo largo de la historia se ha dado a través de cuatro generaciones diferentes, cada una con sus características propias, errores y aciertos que se han tratado de perfeccionar con el pasar de los años. (Rojas, 2016, p. 3)

Por otra parte, el país se encuentra en las vías de cuarta generación las cuales forman parte

[...] de un programa de infraestructura vial establecido en Colombia desde el año 2013, que surge a partir de la asociación entre el sector público y el sector privado, creando agrupaciones contractuales denominadas concesiones, para vincular recursos privados y cuyo objetivo proyecta la construcción, innovación y ampliación de la malla vial del país. (Rojas, 2016, 2016, p. 5)

En cuanto a la malla vial en los municipios,

[...] se utiliza una regla diferente para estimar la brecha, esta depende del tamaño poblacional del municipio. Se busca que las ciudades de más de un millón de habitantes alcancen la proporción de kilómetros de vías principales y troncales del total de kilómetros de la malla vial de Bogotá, que tiene el mayor valor del indicador. (Yepes, Martínez y Aguiar, 2014, p. 11)

Existe una clara necesidad de concretar los planes y acciones en aras de priorizar, de la malla vial, la inmediatez de los requerimientos que exigen la adecuación de las vías, sin importar si son de orden nacional, departamental o municipal.

\section{Materiales y métodos}

La línea de investigación fue la de modelación y simulación de problemas complejos y minería de datos en ingeniería civil. El campo de acción fueron los problemas de movilidad en el municipio de Cachipay y la tipología fue un estudio de caso, que se realizó mediante el análisis e inventario de daños, el planteamiento de mínimo dos alternativas, la selección de la mejor, y el estudio a nivel de fase II de la seleccionada. 
La metodología desarrollada en este trabajo se compone de seis fases:

1. Fase 1. Caracterización del contexto de la situación de la malla vial del municipio de Cachipay en Cundinamarca.

2. Fase 2. Planificación. Se adelantó la programación y se establecieron los recursos directos e indirectos que deparaba el proyecto, en cada una de sus actividades.

3. Fase 3. Adquisición de información primaria y secundaria.

a.De la información primaria. Corresponde a toda aquella información que se requiere levantar o generar de actividades directas en campo:

- Estudio de tráfico. Se establecieron las estaciones de conteo de tráfico; se realizaron los aforos de tráfico vehicular según la normatividad vigente; se establecieron los porcentajes de crecimiento de tráfico para los diferentes tipos de vehículos.

- Se estableció el volumen de tráfico promedio diario anual (TPDA) para el periodo de diseño a 20 años.

- Estudio topográfico. Se realizó con la utilización de equipos de última tecnología que facilitan y agilizan los procesos de modelaciones topográficas, las actividades que requirieron estos trabajos fueron: vuelo fotogramétrico para el mosaico del área de influencia del proyecto; levantamiento topográfico -por sistema convencional, lo que permitió determinar las condiciones topográficas de los corredores existentes, de tal forma se pudo establecer el modelo digital) (superficie del terreno tridimensionalmente)-, y la generación de curvas de nivel de la solución escogida.

- Estudio de geotecnia. Requirió de la investigación del subsuelo mediante apiques en zonas del proyecto, y de los ensayos de laboratorio que permitieron determinar la capacidad de soporte del suelo.

- Inventario de afectaciones a la malla vial del municipio. Involucró el levantamiento de los diferentes tipos de fisuras, deformaciones, pérdida de las capas de la estructura, daños superficiales y otros daños; se determinaron los espesores de las estructuras de pavimentos existentes; se demostró que las vías existentes no estaban diseñadas para el tráfico inesperado de los planes de retorno.

b.De la fuente secundaria. Esta etapa permitió la consulta de información secundaria de la que disponía el municipio de Cachipay, la Gobernación de Cundinamarca, Planeación Nacional, Ministerio de Transporte y todas las entidades oficiales al momento de desarrollar la investigación y las demás que dispongan de información de la normatividad y de estadísticas que puedan relacionarse con este proyecto.

Adicionalmente, se realizó la consecución de información secundaria como cartografía, certificaciones de placas georreferenciadas y de puntos de elevación (NP) del Instituto Geográfico Agustín Codazzi (IGAC), cartografía existente en el municipio de acuerdo con el Esquema de Ordenamiento Territorial (EOT), levantamientos topográficos existentes del municipio, GPS materializados y posicionados, información de volúmenes de tráfico, etc.

4. Fase 4. Procesamiento y análisis de la información primaria. Dentro de las actividades relevantes de esta etapa se pueden mencionar el planteamiento de mínimo dos alternativas de solución para el municipio de Cachipay, a partir de la información secundaria y primaria establecida; realización de la evaluación y selección de la mejor alternativa para el proyecto, a partir, de la matriz de evaluación.

5. Fase 5. Propuesta de solución. Las alternativas de solución ofrecidas por los investigadores son dos, de las cuales se desarrolló la óptima para la solución del problema:

a.Diseños de pavimentos: se diseñó la estructura de pavimento en concreto flexible y en concreto rígido; se hizo la evaluación económica de cada tipo de estructura con precios de referencia o de mercado, y se planteó una recomendación para la estructura apropiada para el proyecto.

b.Diseños geométricos definitivos fase II: se adelantaron los estudios y diseños geométricos definitivos fase II de la solución escogida, lo cual se trazó mediante la utilización del software de AutoCAD Civil 3D 2014.

6. Fase 6. Documento y socialización. 


\section{Resultados}

El desarrollo de las actividades definidas en la metodología permite diagnosticar los efectos generados en la malla vial del municipio de Cachipay, por el tráfico de largo destino generado por los planes retornos impuestos por el Estado. De ese modo, en lo relacionado al estudio de tráfico, los efectos a la malla vial del casco urbano en el municipio de Cachipay, es indispensable desarrollar uno de los alcances del trabajo consistente en la determinación de los volúmenes de tráfico actuales en la zona del proyecto. Por lo anterior, para los conteos de estos se dispuso de cinco cámaras con las que se adelantaron los registros fílmicos durante un periodo de siete días, según la normatividad, y se realizaron los conteos de tráfico, mediante los registros de los tipos de vehículos que circularon durante dicho periodo.

El objetivo general del estudio de tránsito es el realizar los prediseños de las estructuras de pavimento de las vías urbanas, en función de las demandas de tránsito, de manera que garantice el mejoramiento de los niveles de servicio y facilite la movilidad de los usuarios. La información que se recopiló en campo permitió diagnosticar las condiciones actuales de movilidad del municipio, las proyecciones de tránsito y el cálculo de número de ejes equivalentes de 8,2 toneladas, con las metodologías y factores establecidos por Invías.

Conforme a la información recogida, la distribución vehicular porcentual de los vehículos que transitaron en la semana es la que se observa en la tabla 2. Los vehículos de mayor tránsito registraron el 79,7 \%, seguido por los buses y busetas con $10,2 \%$, y camiones, con el restante $10,1 \%$. Entre los camiones, se identificó que el 5,91\% corresponden a C2-P, seguidos por los C2-G con el $3,97 \%$, se evidenció muy bajo volumen vehicular de los $\mathrm{C} 3$ con el 0,02\%, C4 con 0,01\%, C5 con $0,02 \%$. No se evidencia el paso de vehículos de más de cinco ejes.

La tasa de crecimiento establecida para el municipio de Cachipay es del orden del $3 \%$. Por lo anterior, en la tabla 3 se registran en resumen el TPD proyectado desde el año base, a los 10 y 20 años.

Tabla 2. Volumen vehicular por día de la semana

\begin{tabular}{ccccc}
\hline Día & Autos & Busetas-Buses & Camiones & Total \\
\hline Lunes & 1139 & 160 & 160 & 1459 \\
\hline Martes & 1154 & 181 & 187 & 1522 \\
\hline Miércoles & 1074 & 178 & 195 & 1447 \\
\hline Jueves & 1182 & 158 & 157 & 1497 \\
\hline Viernes & 1368 & 150 & 154 & 1672 \\
\hline Sábado & 1646 & 185 & 190 & 2021 \\
\hline Domingo & 2044 & 219 & 180 & 2443 \\
\hline TOTAL & 9607 & 1231 & 1223 & 12061 \\
\hline
\end{tabular}

Fuente: elaboración propia .

Tabla 3. TPD Proyectados de Cachipay

\begin{tabular}{lllllllllll}
\hline \multicolumn{1}{c}{ Periodo } & Año & Livianos & Buses & C 2P & C 2G & C 3 & C 4 & C 5 & >C 5 & TOTAL \\
\hline Año base & 2016 & 1372 & 176 & 102 & 68 & 4 & 0,14 & 0,43 & 0 & 1723 \\
\hline 10 años & 2026 & 1844 & 236 & 137 & 92 & 5 & 0 & 1 & 0 & 2316 \\
\hline 20 años & 2036 & 2479 & 318 & 184 & 124 & 7 & 0 & 1 & 0 & 3,112 \\
\hline
\end{tabular}

Fuente: elaboración propia. 
Teniendo el estudio de tránsito, se realizó el diseño para la estructura de pavimento flexible con la metodología AASHTO 1993 y su verificación por el método racional. Los resultados de los diseños de estructura del pavimento flexible para el tráfico actual y para los periodos de diseño de 10 y 20 años se presentan en la tabla 4.

Tabla 4. Resumen estructuras de pavimento flexible

\begin{tabular}{llllll}
\hline $\mathbf{N}_{\text {80KN de diseño, }}$ & \multicolumn{4}{c}{ Estructura } \\
\hline $\begin{array}{l}\text { Periodo de } \\
\text { diseño }\end{array}$ & $\mathbf{C A}$ & $\mathbf{B G}$ & SBG & Mejoramiento \\
\cline { 2 - 6 } & $\mathbf{C m}$ & $\mathbf{c m}$ & $\mathbf{c m}$ & $\mathbf{c m}$ \\
\hline Actual & 5 & 15 & 30 & 30 & \\
\hline 10 años & 15 & 15 & 30 & 30 & \\
\hline 20 años & 20 & 15 & 30 & 30 \\
\hline
\end{tabular}

Fuente: elaboración propia.

Se desarrolló la alternativa de diseño en pavimento rígido, por medio del método de la Portland Cement Association (PCA). Los resultados de los diseños de estructura del pavimento rígido para el tráfico actual y para un periodo de diseño de 20 años se presentan en la tabla 5 .

Entre las actividades realizadas, se establecieron los espesores de las estructuras de los pavimento flexibles y rígidos existentes, mediante apiques en algunas calles del municipio de Cachipay, estableciendo los espesores y características de los materiales que conforman cada estructura
Tabla 5. Resumen estructuras de pavimento rígido

\begin{tabular}{lccc}
\hline & \multicolumn{3}{c}{ Estructura } \\
\hline $\begin{array}{c}\text { Periodo de } \\
\text { diseño }\end{array}$ & $\mathbf{C H}$ & SBG & Mejoramiento \\
\cline { 2 - 4 } & $\mathbf{c m}$ & $\mathbf{c m}$ & $\mathbf{c m}$ \\
\hline Actual & 20 & 30 & 30 \\
\hline 20 años & 21 & 22.5 & 30 \\
\hline
\end{tabular}

Fuente: elaboración propia.

y la calidad del suelo de la subrasante. Luego, se elaboró un cuadro comparativo entre estos resultados y las estructuras diseñadas para el tráfico y las condiciones de suelos actuales, cuyos resultados permitieron determinar que las estructuras de pavimento existentes no estaban diseñadas o construidas para el tráfico actual, de acuerdo con los ejes equivalentes establecidos en el estudio de pavimentos mediante la información de los conteos de tráfico realizados para el proyecto. A partir del esquema vial del municipio se establecieron desde un diagnóstico inicial una serie de alternativas probables que solucionaran el problema de movilidad que actualmente presenta el municipio de Cachipay, desde dos criterios: manteniendo el paso actual y proyectando una solución que se realizara por vías existentes que implicaran una menor intervención predial, con el fin de lograr una solución inmediata a corto plazo.

Por lo anterior, inicialmente se plantearon cuatro alternativas posibles de solución, pero desde

Tabla 6. Características de las alternativas elegidas para el paso del tráfico de largo destino por el municipio

\begin{tabular}{lll}
\hline \multicolumn{1}{c}{ Criterio } & Alternativa & \multicolumn{1}{c}{ Descripción } \\
\hline $\begin{array}{l}\text { Mantener el tráfico pesado y de largo destino } \\
\text { por la vía actualmente aprobada, paso por el } \\
\text { centro del casco urbano }\end{array}$ & $\begin{array}{l}\text { Primera alternativa } \\
\text { (trazado color negro) }\end{array}$ & $\begin{array}{l}\text { Vía actual que cruza el municipio de Cachipay } \\
\text { de norte a sur por la carrera 3, sector desde El } \\
\text { Progreso hasta Puerto López. Longitud 2,20 km. }\end{array}$ \\
$\begin{array}{l}\text { Proyectar una alternativa para el tráfico } \\
\text { pesado y de largo destino que no cruce por } \\
\begin{array}{l}\text { el centro del casco urbano del municipio } \\
\text { de Cachipay }\end{array}\end{array}$ & $\begin{array}{l}\text { Segunda alternativa } \\
\text { (trazado color rojo) }\end{array}$ & $\begin{array}{l}\text { del municipio de Cachipay, desde el sitio El } \\
\text { Progreso hasta el sitio Puerto López, con una } \\
\text { longitud de 1,74 km. }\end{array}$ \\
\hline
\end{tabular}

Fuente: elaboración propia. 
el análisis de los criterios básicos indicados, finalmente se eligieron dos: la primera, manteniendo el paso actual por el centro del municipio, y la segunda, por el costado oriental del casco urbano.

Posteriormente, se realizaron los diseños geométricos de las dos alternativas seleccionadas, a partir de los criterios y recomendaciones del Instituto Nacional de Vías en el Manual de diseño geométrico de carreteras (2008); las condiciones actuales de la malla vial del municipio y los parámetros definidos en el Esquema de Ordenamiento Territorial (EOT) del municipio, todos como elementos primordiales para los trazados realizados. Los diseños geométricos sobre planta y perfil se integraron mediante el diagrama de peraltes en las secciones transversales, en las cuales se aplicaron los espesores del diseño de pavimento flexible diseñado para el proyecto. El perfil vial utilizado en los diseños geométricos para cada alternativa elegida está acorde con lo establecido en dicho EOT.

Una vez elaborados los diseños geométricos, y a partir de la información suministrada en el municipio, se hizo una evaluación comparativa a través de una metodología multicriterio, por medio de la cual se escogieron cuatro alternativas para la solución de la movilidad en el municipio de Cachipay, siguiendo los lineamientos de los criterios de evaluación.

Cada criterio seleccionados contempla subcriterios que lo fortalecen, según se indica a continuación: criterio de diseño geométrico, volúmenes de corte, volúmenes de materiales de construcción y longitud total de la alternativa; criterio del medio físico o abiótico, geología y geotecnia, amenazas por remoción en masa, e hidrología cantidad de cruces de quebradas y ríos; criterios del medio biótico: coberturas vegetales y especies faunísticas, y criterio del medio socioeconómico y cultural: población beneficiada por el proyecto, costos alternativos, adquisición predial, dificultad movilidad vehicular y funcionalidad peatonal.

Por cada uno de los subcriterios se realizó la comparación según las cantidades obtenidas en cada alternativa y para calcular el valor ponderal; se sumaron ambos valores y se estableció el porcentaje que cada alternativa representaba sobre dicho total; posteriormente se estableció el mayor puntaje a la alternativa más deseable para el proyecto.

El análisis jerárquico se realizó mediante la construcción de una matriz pareada de criterios (tabla 7). Cada celda superior a la diagonal dio lugar a la valoración de cada par de criterios: el de la fila versus el de la columna. Se emitió juicio de valor respecto a la contundencia entre uno y otro criterio, respecto al estudio y selección de alternativa en especial.

Efectuada la valoración a través de la expresión de juicios de valor, se obtuvo una matriz normalizada (tabla 8). En consecuencia, se sumaron las columnas de la matriz anterior (matriz de comparación pareada) y cada valor de celda fue dividido

Tabla 7. Comparación pareada de criterios

\begin{tabular}{lccccc}
\hline \multicolumn{1}{c}{ Criterios } & $\begin{array}{c}\text { Diseño } \\
\text { geométrico }\end{array}$ & $\begin{array}{c}\text { Medio } \\
\text { físico }\end{array}$ & $\begin{array}{c}\text { Medio } \\
\text { biótico }\end{array}$ & $\begin{array}{c}\text { Medio socioeconómico } \\
\text { y cultural }\end{array}$ & $\begin{array}{c}\text { Promedio de } \\
\text { fila }\end{array}$ \\
\hline Diseño geométrico & 1,00 & 0,25 & 0,20 & 3,00 & 1,113 \\
\hline Medio físico & 4,00 & 1,00 & 0,50 & 5,00 & 2,625 \\
\hline Medio biótico & 5,00 & 2,00 & 1,00 & 4,00 & 3,000 \\
\hline Medio socioeconómico y cultural & 0,33 & 0,20 & 0,25 & 1,00 & 0,446 \\
\hline Suma de columna & 10,33 & 3,45 & 1,95 & 13,00 & - \\
\hline
\end{tabular}

Fuente: elaboración propia. 
por el total obtenido (suma de columnas). Esto se repitió para cada columna, obteniendo un promedio por fila.
En la tabla 9 se presenta la comparación por pares de los criterios del diseño geométrico y en la tabla 10 se presenta la normalización de los valores.

Tabla 8. Matriz principal normalizada

\begin{tabular}{lccccc}
\hline \multicolumn{1}{c}{ Criterios } & $\begin{array}{c}\text { Diseño } \\
\text { geométrico }\end{array}$ & Medio físico & $\begin{array}{c}\text { Medio } \\
\text { biótico }\end{array}$ & $\begin{array}{c}\text { Medio } \\
\text { socioeconómico y } \\
\text { cultural }\end{array}$ & $\begin{array}{c}\text { Promedio } \\
\text { de fila }\end{array}$ \\
\hline Diseño geométrico & 0,097 & 0,072 & 0,103 & 0,231 & 0,126 \\
\hline Medio físico & 0,387 & 0,290 & 0,256 & 0,385 & 0,329 \\
\hline Medio biótico & 0,484 & 0,580 & 0,513 & 0,308 & 0,471 \\
\hline Medio socioeconómico y cultural & 0,032 & 0,058 & 0,128 & 0,077 & 0,074 \\
\hline Suma de columna & 1,00 & 1,00 & 1,00 & 1,00 & 1,00 \\
\hline
\end{tabular}

Fuente: elaboración propia.

Tabla 9. Comparación pareada de criterios del diseño geométrico

\begin{tabular}{lcccc}
\hline \multicolumn{1}{c}{ Criterios } & $\begin{array}{c}\text { Volúmenes de } \\
\text { corte }\end{array}$ & $\begin{array}{c}\text { Volúmenes de } \\
\text { materiales de } \\
\text { construcción }\end{array}$ & $\begin{array}{c}\text { Longitud total } \\
\text { de la alternativa }\end{array}$ & Promedio de fila \\
\hline Volúmenes de corte & 1,00 & 2,00 & 3,00 & 2,000 \\
\hline $\begin{array}{l}\text { Volúmenes de materiales de } \\
\text { construcción }\end{array}$ & 0,50 & 1,00 & 4,00 & 1,833 \\
\hline Longitud total de la alternativa & 0,33 & 0,25 & 1,00 & 0,527 \\
\hline Suma de columna & 1,83 & 3,25 & 8,00 & - \\
\hline
\end{tabular}

Fuente: elaboración propia.

Tabla 10. Matriz normalizada para los criterios del diseño geométrico

\begin{tabular}{lcccc}
\hline \multicolumn{1}{c}{ Criterios } & $\begin{array}{c}\text { Volúmenes de } \\
\text { corte }\end{array}$ & $\begin{array}{c}\text { Volúmenes de } \\
\text { materiales de } \\
\text { construcción }\end{array}$ & $\begin{array}{c}\text { Longitud } \\
\text { total de la } \\
\text { alternativa }\end{array}$ & $\begin{array}{c}\text { Promedio de } \\
\text { fila }\end{array}$ \\
\hline Volúmenes de corte & 0,546 & 0,615 & 0,375 & 0,512 \\
\hline Volúmenes de materiales de construcción & 0,273 & 0,308 & 0,500 & 0,541 \\
\hline Longitud total de la alternativa & 0,181 & 0,077 & 0,125 & 0,128 \\
\hline Suma de columna & 1,00 & 1,00 & 1,00 & - \\
\hline
\end{tabular}

Fuente: elaboración propia. 
En la tabla 11 se presenta la comparación por pares de los criterios del medio físico, y en la tabla 12, la normalización de los valores.
En la tabla 13 se presenta la comparación por pares de los criterios del medio biótico y en la tabla 14, la normalización de los valores.

Tabla 11. Comparación pareada de criterios del medio físico

\begin{tabular}{lccc}
\hline \multicolumn{1}{c}{ Criterios } & $\begin{array}{c}\text { Vulnerabilidad de } \\
\text { la vía a procesos de } \\
\text { inestabilidad }\end{array}$ & $\begin{array}{c}\text { Cruces de } \\
\text { quebradas y } \\
\text { ríos }\end{array}$ & Promedio de fila \\
\hline Vulnerabilidad de la vía a procesos de inestabilidad & 1,00 & 5,00 & 3,000 \\
\hline Cruces de quebradas y ríos & 0,20 & 1,00 & 0,600 \\
\hline Suma de Columna & 1,20 & 6,00 & - \\
\hline
\end{tabular}

Fuente: elaboración propia.

Tabla 12. Matriz normalizada para los criterios del medio físico

\begin{tabular}{lccc}
\hline Criterios & $\begin{array}{c}\text { Vulnerabilidad de } \\
\text { la vía a procesos de } \\
\text { inestabilidad }\end{array}$ & $\begin{array}{c}\text { Cruces de } \\
\text { quebradas y ríos }\end{array}$ & Promedio de fila \\
\hline Vulnerabilidad de la vía a procesos de inestabilidad & 0,833 & 0,833 & 0,833 \\
\hline Cruces de quebradas y ríos & 0,167 & 0,167 & 0,167 \\
\hline Suma de Columna & 1,00 & 1,00 & - \\
\hline
\end{tabular}

Fuente: elaboración propia.

Tabla 13. Comparación pareada de criterios del medio biótico

\begin{tabular}{lccc}
\hline \multicolumn{1}{c}{ Criterios } & Coberturas vegetales & Especies faunísticas & Promedio de fila \\
\hline Coberturas vegetales & 1,00 & 4.00 & 2.50 \\
\hline Especies faunísticas & 0.25 & 1,00 & 0,625 \\
\hline Suma de columna & 1,25 & 5,00 & - \\
\hline
\end{tabular}

Fuente: elaboración propia.

Tabla 14. Matriz normalizada para los criterios del medio biótico

\begin{tabular}{lccc}
\hline \multicolumn{1}{c}{ Criterios } & Coberturas vegetales & Especies faunísticas & Promedio de fila \\
\hline Coberturas vegetales & 0.800 & 0,800 & 0,800 \\
\hline Especies faunísticas & 0.200 & 0,200 & 0,200 \\
\hline Suma de columna & 1,00 & 1,00 & - \\
\hline
\end{tabular}

Fuente: elaboración propia. 
Posteriormente se realizó la comparación por pares de los criterios del medio socioeconómico y cultural, a través de la normalización de los valores, Ilevando a la ponderación por componentes y criterios, para fijar la opción más apropiada para el municipio de Cachipay.

\section{Discusión}

La búsqueda de soluciones a los problemas de movilidad en el municipio de Cachipay por los planes de retorno que el Estado ha venido implementando para agilizar la movilidad vehicular entre el municipio de La Mesa y la ciudad de Bogotá D.C., ha generado un incremento de circulación vehicular por las calles del municipio, lo cual se pudo establecer según los conteos de tráfico registrados en los días entre semana contra el día festivo, cuyo aumento oscila entre un $67 \%$ a un $46 \%$, como se registra en la tabla 15.

Los efectos generados por el tráfico de largo destino en la malla vial del municipio de Cachipay son el evidente incremento de volúmenes de tráfico en las vías del municipio, en los fines de semana; incremento del orden del $52 \%$ en la ocupación de las vías del municipio autorizadas para el tráfico de largo destino.

El inventario de daños, realizado sobre las vías ocupadas por tráfico de largo destino, evidenció una serie de daños: fisuras, deformaciones, pérdidas de las capas de las estructuras de los pavimentos, daños superficiales y afloramientos de materiales y de agua; todos estos afectan la movilidad vehicular.

Se estableció, a partir de los conteos de tráfico, que las estructuras de pavimento flexible y rígido existentes no eran apropiadas para los volúmenes de tráfico actuales. Estas estaban previstas para solo un $50 \%$ en el caso de pavimentos flexibles y un $75 \%$ para el caso de pavimentos rígidos.

Otros aspectos evidenciados, y que afectan la movilidad en el municipio, fueron: el desorden en el comportamiento de los usuarios en las vías y de los espacios públicos -como parquear a lado y lado de estas-, ocupación de los espacios públicos para actividades comerciales, movilización peatonal por las calzadas de las vías producto de la ocupación de los espacios públicos.

\section{Conclusiones y recomendaciones}

Se carece de una infraestructura adecuada que garantice una trazabilidad apropiada, como zonas o espacios adecuados para el servicio de parqueo y una señalización conveniente en las áreas de vías municipales.

El problema de movilidad en el interior del municipio requiere de una solución pronta y efectiva, de lo cual se deriva una clara necesidad de reformar la malla vial de largo destino en el municipio de Cachipay. Así, se logrará una mayor conectividad intermunicipal y se generará un crecimiento dentro del municipio.

Tabla 15. Planes de retorno

\begin{tabular}{lcccccc}
\hline \multicolumn{1}{c}{ Día } & Autos & Busetas-Buses & Camiones & Total & $\begin{array}{c}\text { Tráfico Domingo vs. } \\
\text { Tráfico día de la semana }\end{array}$ & Incremento (\%) \\
\hline Lunes & 1139 & 160 & 160 & 1459 & $167 \%$ & $67 \%$ \\
\hline Martes & 1154 & 181 & 187 & 1522 & $161 \%$ & $61 \%$ \\
\hline Miércoles & 1074 & 178 & 195 & 1447 & $169 \%$ & $69 \%$ \\
\hline Jueves & 1182 & 158 & 157 & 1497 & $163 \%$ & $63 \%$ \\
\hline Viernes & 1368 & 150 & 154 & 1672 & $146 \%$ & $16 \%$ \\
\hline Sábado & 1646 & 185 & 190 & 2021 & $100 \%$ & \\
\hline Domingo & 2044 & 219 & 180 & 2443 & 100 & \\
\hline
\end{tabular}

Fuente: elaboración propia. 
Teniendo claro que la reforma de la malla vial debe ser inminente, el estudio realizado planteó inicialmente cuatro alternativas por rutas que ofrecieran una implementación con las menores intervenciones. De estas, se establecieron dos alternativas viables que ofrecen una panorámica completa de las posibilidades que se pueden o no tener en cuenta, seleccionando la más apropiada mediante una matriz multicriterio. Se puede señalar, entonces, que la más apropiada es aquella que trascurre por área urbana por el costado oriental del municipio.

La situación actual de la malla vial en el municipio de Cachipay constituye un derrotero para el país, en materia de infraestructura de transporte y movilidad; razón por la cual, estudios como el este resultan pertinentes y apropiados en la consecución de alternativas de solución vial.

Cachipay, por ser un municipio cercano a la capital, debe contar con propuestas que contribuyan a solucionar la problemática en el deterioro de las calles y vías alternas para fortalecer el parque automotor del municipio.

Del trabajo realizado es importante indicar una serie de recomendaciones que deben ser implementadas y que pueden contribuir a soluciones en otros municipios afectados por las mismas circunstancias establecidas en este trabajo, entre estas podemos indicar:

- Implementar zonas o espacios apropiados para parqueaderos, con planes de recaudo que permitan la obtención de recursos que sirvan a las inversiones requeridas.

- Implementar planes o programas de capacitaciones, o educación vial, de tal forma que se establezcan las estrategias en pro del control eficiente del tráfico vehicular de motorizados que fluyen por las vías, así como sincronizar dicho tráfico junto con el de transeúntes (no motorizados y sin placas de circulación) cuyos desplazamientos coinciden inevitablemente $y$ hacen necesario un proceso permanente de aprendizaje e instrucción en materia de seguridad vial y cultura vial.

- Proceder con la recuperación del espacio público, que actualmente se encuentra afectado por el comercio de los negocios aledaños a las vías.

- Continuar con el desarrollo de estudios similares que permitan lograr una cultura vial, por lo menos de carácter departamental, dado que gran número de municipios aledaños a la capital presentan los mismos problemas.

- Tener en cuenta el presente estudio para desarrollarlo.

- Continuar con esta clase de investigaciones como una forma de contribuir con la responsabilidad social.

\section{Referencias}

Alcántara, V. (2010). Análisis de la movilidad urbana. Espacio, medio ambiente y equidad. Bogotá, D.C.: Corporación Andina de Fomento.

Concejo Municipal de Cachipay. Acuerdo 04 del 4 de abril de 2008. Por el cual se adopta el plan de desarrollo para el municipio de Cachipay "sembrando desarrollo social" para la vigencia 2008-2012 y se dictan otras disposiciones. Cachipay.

Gaviria M., S. (2016). Diálogos regionales para la planeación de un nuevo país. Bogotá, D.C.: Departamento Nacional de Planeación.

Gobernación de Cundinamarca (2009). Plan Vial del Departamento de Cundinamarca. Bogotá, D.C.: Secretaría de Transporte y Movilidad.

Instituto Nacional de Vías (Invías) (2008). Manual de diseño geométrico de carreteras. Bogotá, D.C.

Pachón, A y Ramírez, M. (2006). La infraestructura de transporte en Colombia durante el siglo XX. Bogotá, D.C.: Fondo de Cultura Económica.

Rey, A. (2016). Plan de desarrollo Cundinamarca "Unidos podemosmás 2016-2020". Recuperadodewww.cundinamarca.gov.co: http://www.cundinamarca.gov. co/wcm/connect/2a9dd7d1-d693-414a-94cd-37fe$5 f 901 \mathrm{e} 7 \mathrm{~d} / \mathrm{PLAN}+\mathrm{DE}+\mathrm{DESARROLLO+VERSION+FI-}$ NAL.pdf?MOD=AJPERES\&CVID=IDIW39U 
Rojas, D. (2016). Desarrollo vial en Colombia y el impacto de las vías de cuarta generación. Bogotá, D.C.: Universidad Militar Nueva Granada.

Yepes, T., Martínez, S. y Aguilar, J. (2014). Financiación de la infraestructura en los municipios del sistema de ciudades de Colombia. Bogotá, D.C.: Fedesarrollo.

Zamora, N. (2012). Diagnóstico de la infraestructura vial actual en Colombia. Bogotá, D.C.,: Universidad EAN.

\section{(c)}

